\title{
Assessment of cardiotoxicity in hormone positive postmenopausal breast cancer patients receiving aromatase inhibitors
}

\author{
Reham M. Faheim, Eman A. El-Shaarawy, Dina A. Salem, Rehab G. Shaaban \\ Clinical Oncology and Nuclear Medicine, Faculty of Medicine, Ain Shams University, Cairo, Egypt
}

Received: January 9, 2019

Accepted: January 10, 2019

Online Published: January 16, 2019

DOI: $10.5430 /$ jst.v9n1p11

URL: https://doi.org/10.5430/jst.v9n1p11

\begin{abstract}
Background: Aromatase inhibitors (AIs) represent an effective endocrine treatment for hormone receptor-positive postmenopausal breast cancer patients with early stage or metastatic disease.

Objective: Assessment of Cardiotoxicity in Hormone positive Postmenopausal Breast Cancer Patients receiving AIs (upfront or switch therapy).

Methods: This cross sectional study included 123 postmenopausal breast cancer patients presented to the Clinical Oncology Department, Ain Shams University (Cairo, Egypt) in the interval from August 2016 to June 2017 with hormone receptor positive receiving Aromatase Inhibitors, To assess cardiotoxicity in these patients, they were subjected to blood pressure and lipid profile measurement, electrocardiography (ECG), and electrocardiography (ECHO) and classified into patients had Nolvadex then A.I (arm 1) and others had upfront A.I (arm 2).

Results: The age of patients ranged from 41 years to 85 years with mean age of 61 years. Seventy one patients (57.7\%) showed cardiotoxicity as assessed by ECHO. They showed significant correlation with rising age above 62 years, IHD, history of HTN and DM ( $p$ value: $.001, .001, .017$ and 0.035 respectively). However, correlation between cardiotoxity and blood pressure changes, lipid profile changes and ECG findings and ECHO changes in switch therapy and upfront A.I were not statistically significant $(p$ value $=.275, .116, .081$ and .761 respectively).

Conclusion: Assessment of cardiotoxicity in hormone positive postmenopausal breast cancer patients receiving Aromatase Inhibitors showed evidence of cardiotoxicity in half the patients (57.7\%) as detected by ECHO only. They showed statistically non significant correlations either recievied switch therapy or upfront A.I.
\end{abstract}

Key Words: Cardiotoxicity, Hormone positive breast cancer, Postmenopausal, Aromatase inhibit

\footnotetext{
${ }^{*}$ Correspondence: Reham M. Faheim; Email: rehamfaheim14@gmail.com; Address: Clinical Oncology and Nuclear Medicine, Faculty of Medicine, Ain Shams University, Cairo, Egypt.
} 